\title{
A Simple Technique to Prevent External Jugular Vein Compression after Microanastomosis
}

\author{
${ }^{1}$ Prabha S Yadav, ${ }^{2}$ Quazi G Ahmad, ${ }^{2}$ Vinay K Shankhdhar, ${ }^{3} \mathrm{GI}$ Nambi \\ ${ }^{1}$ Professor and Head, Plastic and Reconstructive Services, Department of Surgical Oncology, TATA Memorial Hospital, Parel \\ Mumbai, Maharashtra, India \\ ${ }^{2}$ Assistant Professor, Plastic and Reconstructive Services, Department of Surgical Oncology, TATA Memorial Hospital, Parel \\ Mumbai, Maharashtra, India \\ ${ }^{3}$ Fellow (Reconstructive Microsurgery), Plastic and Reconstructive Services, Department of Surgical Oncology, TATA Memorial \\ Hospital, Parel, Mumbai, Maharashtra, India
}

Correspondence: GI Nambi, Fellow (Reconstructive Microsurgery), Plastic and Reconstructive Services, Department of Surgical Oncology, TATA Memorial Hospital, Parel, Mumbai-400012, Maharashtra, India, e-mail: Nambi75@rediffmail.com Phone: $+91-9619746087$

\section{Abstract}

External jugular vein (EJV) is one of the most commonly used veins for microanastomosis in head and reconstruction. Being in the subcutaneous plane, the EJV is prone for compression from both external and internal factors after mobilization for anastomosis. To prevent this, we describe a technique where a trench is created in the sternomastoid muscle in which the EJV is placed safely and prevented from compression.

Keywords: Head and neck reconstruction, microsurgery, anastomosis, external jugular vein, sternomastoid trench.

The external jugular vein (EJV) is one of the most commonly used veins for microanastomosis in reconstruction of the head and neck structures. The other veins being the various tributaries of the internal jugular vein (IJV) and the IJV itself. Whereas, the EJV and the tributaries of the IJV are used for end-to-end (E-E) anastomosis, the IJV is used for end-to-side (E-S) anastomosis with the flap vein.

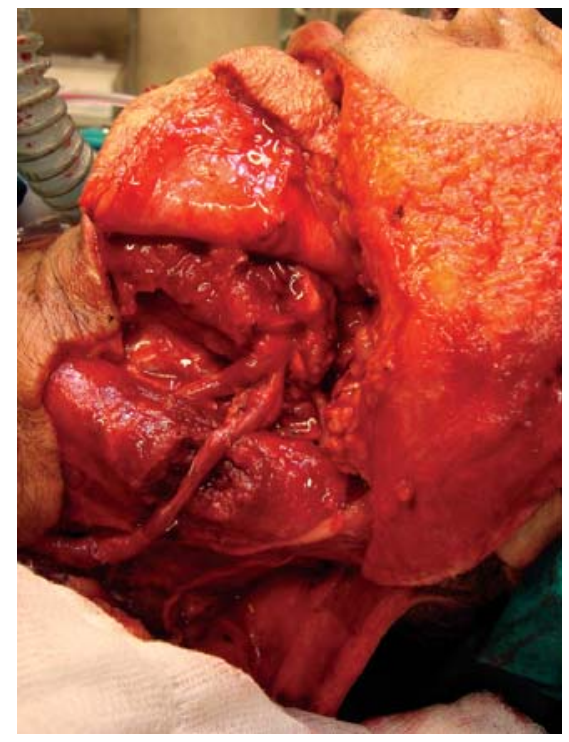

Fig. 1: The external jugular vein is safely placed in the sternomastoid trench
In microsurgery, mobilization of the external jugular vein for anastomosis may result in distortion or kinking of the vessel and prone compression by external or internal factors. The external factor is the edema of the neck skin flaps raised during various types of lymph node dissections resulting in tight closure of the neck wound. Depending up on the type of neck node dissections, the sternomastoid muscle may be retained or removed. If retained, edema of the sternomastoid

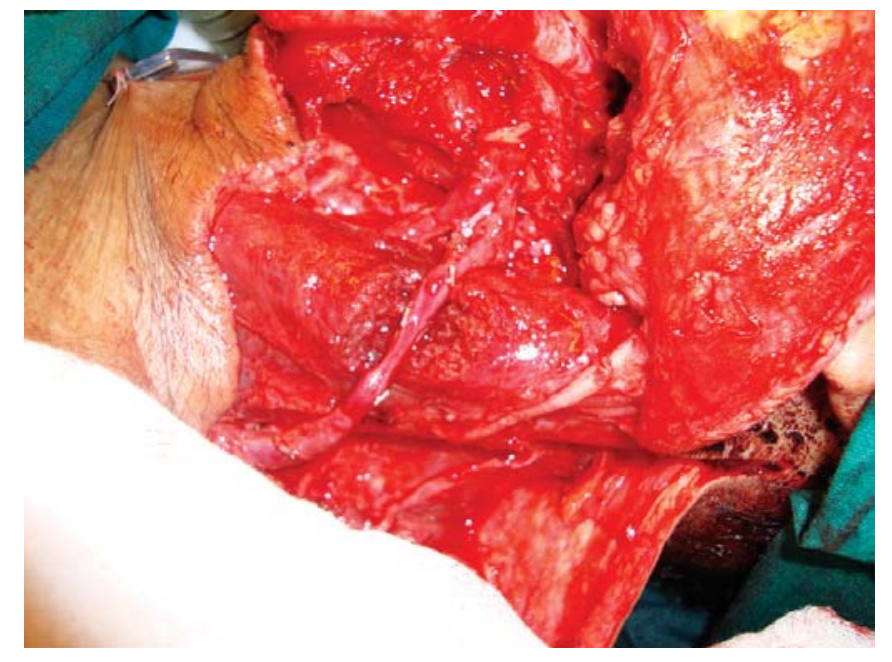

Fig. 2: Closer view 
muscle contributes for the compression of the EJV from inside. Severity of one or both of these factors may result in compression of the EJV resulting in poor venous drainage of the flap. In order to avoid this we create a trench in the sternomastoid muscle (Figs 1 and 2) by dividing the superficial fibers across the muscle sufficient enough to place the EJV. This protects the EJV from the compressive factors without compromising the function of the sternomastoid muscle. This technique is helpful when only a single vein (EJV) is used for microsurgical reconstruction of the head and neck structures following tumor ablative surgeries. 